\title{
Educación jurídica y educación democrática en América Latina: Las revistas jurídicas dirigidas por estudiantes de grado, la experiencia comparada y un caso de estudio argentino: la Revista Lecciones y Ensayos
}

\author{
Educação jurídica e educação democrática na América Latina: revistas jurídicas \\ dirigidas por estudantes de graduação, a experiencia comparada e um estudo de caso \\ argentino, a Revista Lecciones y Ensayos
}

Legal education and democractic education in Latin America: Student-run law reviews, the comparative experience and an Argentine case study, Lecciones y Ensayos magazine

\author{
Patricio Enrique Kenny \\ Universidad de Buenos Aires y Universidad de San Andrés, Argentina
}

\begin{abstract}
RESUMEN En este artículo se abordan las principales discusiones que tienen relación con las revistas jurídicas dirigidas exclusivamente por estudiantes de grado. Al mismo tiempo, se pretende incorporar nuevas perspectivas propias del contexto latinoamericano y, en especial, argentino, a estos debates. Por último, se incluyen algunos desafíos para los próximos años en lo que respecta al objeto de estudio. Todo esto puede ser de utilidad para quienes deseen continuar el debate sobre las revistas jurídicas latinoamericanas y, en particular, a aquellas dirigidas exclusivamente por estudiantes de grado.
\end{abstract}

PALABRAS CLAVE Revistas dirigidas por estudiantes de grado, democracia, educación, América Latina.

RESUMO Neste artigo, revisa-se uma das principais discussões que vêm ocorrendo em relação a periódicos jurídicos dirigidos exclusivamente por estudantes de graduação. Ao mesmo tempo, pretendemos incorporar novas perspectivas próprias do contexto latino-americano, especialmente da Argentina, a esses debates. Finalmente, alguns possíveis desafios para os próximos anos estão incluídos no estudo de caso escolhido. Tudo isso pode ser útil para aqueles que desejam continuar a discutir revistas jurídicas 
latino-americanas e, em particular, aquelas dirigidas exclusivamente por estudantes de graduação.

PALAVRAS-CHAVE Revistas dirigidas por estudantes, democracia, educação, América Latina.

\begin{abstract}
In this article, I describe a some of the main discussion that have been taking place so far in relation to student-run law reviews. At the same time, I pretend to mention some new perspectives, based on the Latin American experience -and especially on the Argentine experience- that may enrich current debates. Finally, I refer to some possible challenges for my case study for the coming years. I believe that all these might be useful for those who wish to become involved in the debates concerning law reviews and, in particular, to student-run law reviews.
\end{abstract}

KEYWORDS Student-run law reviews, democracy, education, Latin America.

\title{
Introducción
}

El debate sobre la incidencia de las revistas dirigidas por estudiantes en la educación jurídica en América Latina es escaso. Lo cierto es que existen pocas revistas latinoamericanas cuyos consejos editoriales se conformen exclusivamente por estudiantes de grado. Muchas de estas revistas fueron ideadas inspirándose en lo que sucede en los Estados Unidos, donde desde 1875 han proliferado, inicialmente de un modo muy informal, los llamados student-run law reviews. (Harper, 1998: 1.263; Movsesian, 2005: 1.112).

Este artículo se ocupará del caso de Lecciones y Ensayos de la Facultad de Derecho de la Universidad de Buenos Aires, una revista jurídica argentina que se encuentra dirigida solo por estudiantes de grado. Considero importante que el debate público pueda extenderse a este espacio, ya que este tipo de revistas no solo repercuten sobre la calidad de la educación superior (Álvarez, Sandoval y Puello, 2018; Bezanilla Albisua y otros, 2018; Carrillo, 2011; Casquero Oyarzabal y otros, 2014; De Mello y Pamplona, 2015; De la Fuente y otros, 2010; García y Lorente, 2017; Gómez y otros, 2018), sino que además signan el perfil de quienes tendrán injerencia en asuntos comunitarios y, no menos importante, suelen hacer uso de fondos públicos.

Para lograr lo anterior se considerarán, en primer lugar, las discusiones pasadas que se han dado en torno de las revistas dirigidas exclusivamente por estudiantes de grado. La experiencia anglosajona, por la abundancia de sus registros, puede ser de utilidad para diseñar alternativas de superación que se ajusten a la realidad latinoamericana. En segundo lugar, se incorporarán nuevas consideraciones propias del contexto argentino, ya que su experiencia puede ofrecer nuevas perspectivas para 
los futuros debates. En particular, me concentraré en cómo estos espacios podrían repercutir favorablemente en la educación jurídica y en la educación cívica de los estudiantes. Estas nuevas perspectivas traen consigo lo que intentan ser expresiones de un ideal regulativo medianamente factible. En tercer lugar, se incluirán posibles desafíos para los próximos años en lo que respecta al objeto de estudio. Sin duda, este recorrido puede ser de utilidad para quienes deseen involucrarse en los debates que se están desarrollando actualmente sobre las revistas jurídicas latinoamericanas.

Para comenzar, es importante mencionar que en 1834 se fundó la primera revista jurídica en la Argentina, El Correo Judicial, que solo pudo editar ocho números debido a la reticencia por parte de los jueces a suministrar las sentencias que pretendían ser publicadas para evitar comentarios o reproches (Pugliese, 2015: 81). Dentro de la Facultad de Derecho de la Universidad de Buenos Aires, entre las primeras revistas se encuentran los Anales de la Facultad de Derecho (1902) y la Revista de la Facultad de Derecho y Ciencias Sociales (1922), en las que se observa una marcada influencia de las revistas de Francia, Italia, España y otros países europeos (Pugliese, 2007: 32; Pugliese, 2015: 82).

A su vez, la revista Lecciones y Ensayos fue fundada en el año 1956 por Ignacio Winizky, director del Departamento de Publicaciones de la Facultad de Derecho de la Universidad de Buenos Aires. En ese momento, luego de que en 1955 se produjera un nuevo golpe de Estado en la Argentina, Luis M. Baudizzone era el decano interventor. Como revista jurídica dirigida exclusivamente por estudiantes de grado, logró mantenerse durante más de 60 años en un contexto de dictaduras, cesantía y censuras.

La experiencia norteamericana ejerció una considerable influencia en la revista, lo que puede explicarse a partir del hecho de que Winizky fue profesor visitante en universidades norteamericanas. En efecto, el surgimiento de los student-run law reviews en los Estados Unidos tuvo repercusiones en distintas partes del mundo como Inglaterra, México, Australia, Canadá y Nueva Zelanda, entre otros países, dentro de los cuales se encuentra Argentina (Closen y Dzielak, 1996: 40). Es importante mencionar que algunos modelos similares a la revista Lecciones y Ensayos pueden encontrarse, por ejemplo, en Brasil, con la Revista do Centro Acadêmico Afonso Pena; en Perú, con la revista Themis; y en Puerto Rico, con la Revista Jurídica de la Universidad de Puerto Rico (Delgado Cintrón, 1982: 4).

\section{Algunas críticas a las revistas jurídicas dirigidas por estudiantes de grado}

El modelo de revistas dirigidas exclusivamente por estudiantes de grado se encuentra presente mayoritariamente en la educación jurídica. En otras disciplinas solo existen, por lo general, publicaciones académicas con examinadores externos que no son estudiantes, sino académicos formados (Allen y Maheandiran, 2009: 1.163-1.164). De hecho, que un académico del derecho publique en alguna de las revistas importantes 
dirigidas por estudiantes en los Estados Unidos puede suponer un mayor prestigio que centrarse en el modelo tradicional de revista con evaluadores externos. Además, en dicho país las revistas dirigidas por estudiantes son las que abundan y las otras escasean (Stracher, 2007-2008: 351).

Desde hace varias décadas, se han formulado innumerables objeciones a la idea de contar con revistas jurídicas dirigidas solo por estudiantes de grado dentro de las universidades (Posner, 1995: 1.131; Rodell, 1936: 38). Las principales críticas apuntan contra el elitismo en la composición de sus cuerpos editoriales y la consiguiente exclusión de los grupos marginados de la comunidad universitaria, la innecesaria complejidad de los sistemas de citación y la inutilidad de estas revistas para la sociedad. Por otra parte, también se critica el sistema arbitrario de selección de los artículos, la demora en su publicación, la falta de una devolución fundada ante un rechazo a publicar un determinado artículo y la falta de anonimato a la hora de evaluar los artículos. Sin embargo, la ausencia de valor pedagógico de estas revistas para los estudiantes editores y los costos operativos de las publicaciones también son factores a considerar a propósito de esta reflexión. A continuación, me detendré en algunos de estos aspectos.

\section{Elitismo}

Las revistas en Estados Unidos suelen estar conformadas por un reducido número de estudiantes seleccionados a partir de las calificaciones obtenidas en su primer año de estudio, es decir, luego de haber terminado el college. Así, habría una tensión entre este sistema y un modelo de educación inclusivo (Klinger, 2015: 184). A partir de la década de los sesenta, y como una reacción antielitista, se empezó a matizar este criterio de selección, incluso hasta llegar a suprimirlo e incorporar otros elementos, por ejemplo, la presentación de un trabajo escrito (Sloviter, 2002: 11). En sus orígenes, Lecciones $y$ Ensayos solo aceptaba a quienes tuviesen calificaciones superiores a «buenas» y estuviesen cursando los dos últimos años de la carrera.

\section{Grupos desaventajados}

Quizás como corolario del elitismo, los cuerpos editoriales de las revistas jurídicas en Estados Unidos no acostumbran a reflejar la diversidad presente en la población universitaria (Knize, 2013: 311). Sin duda, suele existir una disparidad de género (Mullins y Leong, 2011: 432) y la participación tanto de mujeres (Grosenick, 2013: 306) como de afroamericanos tiende a ser reducida (Smith, 2011: 82). Además, las minorías que desean publicar son silenciadas indirectamente, ya que los artículos son examinados mayoritariamente por estudiantes editores varones y blancos (Godsey, 1995: 70).

Por otro lado, la dinámica de estos espacios a veces puede conducir a que sus 
integrantes adopten una impronta de opresión a la diferencia. Como explican Knize (2013: 325) y Sturm (1997: 121-122) a través del modelo de gladiadores, primero puede enfatizarse la competencia por sobre la colaboración, que se manifiesta, entre otras cosas, en el trabajo editorial en general, la evaluación de los artículos recibidos y la selección de los cargos electivos. Segundo, este modelo se centra en las reglas y las estructuras jerárquicas en lugar de las relaciones interpersonales. Esto conduce a que los que presentan mayores sentimientos de empatía y flexibilidad se sientan alienados (Kymlicka, 1995: 287). Tercero, el modelo de gladiadores pareciera promover la consolidación de futuros abogados con perfiles que refuerzan el diseño de las estructuras institucionales predominantes: el verticalismo, el secretismo de lo público, el autoritarismo. Estos tres aspectos, de constatarse, pueden traer graves consecuencias para la participación de los sectores más marginados de la comunidad universitaria.

\section{Utilidad}

Muchas veces se ha dicho que el contenido de estas revistas no posee ninguna importancia, es decir, que es de poca utilidad. Además, se añade que su contenido no suele ser equilibrado en cuanto a artículos filosóficos y prácticos (Fowler, 2001: 49). Por otra parte, se asevera que son muy aburridos, pesados, tediosos y que su estilo es poco atractivo (Edwards, 2014: 1488). A todo esto se suma, según la opinión de algunos, el hecho de que actualmente los académicos se ven obligados a publicar frenéticamente para poder sobrevivir y que, por consiguiente, el resultado puede ser de baja calidad en muchas sus producciones (Gava, 2002: 576).

\section{Selección de artículos}

En reiteradas ocasiones se ha sostenido que los estudiantes carecen de suficiente conocimiento y experiencia para seleccionar los mejores artículos, circunstancia que se vería exacerbada por la creciente interdisciplinariedad del derecho (Posner, 1995: 1.133-1.134). A esto se le agrega que en Estados Unidos, por lo general, cada dos años la composición del cuerpo editorial se modifica por completo. Así, es difícil que sus miembros puedan adquirir los suficientes conocimientos antes de abandonar la revista y no solo se afecta la memoria institucional, sino también los proyectos a largo plazo (Wise y otros, 2013: 8).

Por otro lado, hay quienes sostienen que los artículos que se envían abordan tantas áreas del derecho y otras disciplinas que hay bajas probabilidades de que un académico formado posea un mayor conocimiento que los estudiantes. De esta forma, los estudiantes serían los que en mejor posición se encuentran para decidir la publicación de un artículo porque son generalistas, ya que se han familiarizado con una gran diversidad de áreas del derecho, lo que se contrapone a la especialización 
exacerbada que hoy pareciera caracterizar a la formación de los académicos (Cotton, 2006; Martineau, 2001). Además, siempre existe la posibilidad de que los estudiantes, ante la duda, recurran a la opinión de los colaboradores expertos. Se argumenta que un artículo que tenga sentido para un estudiante editor probablemente también tendrá sentido para el que, habiéndose especializado en un área en particular, desea comprender un artículo de otra área (Nichols, 1987: 1.130).

\section{Demora y falta de fundamentos ante el rechazo}

Se puntualiza que el trabajo de los estudiantes editores es demasiado lento a la hora de publicar los artículos. Además, generalmente las revistas jurídicas no ofrecen a los autores los motivos fundados por los cuales se rechazó la obra y tampoco sugieren las formas en que puede ser mejorada (Wise y otros, 2013: 18). En respuesta, se esgrime que proporcionar una devolución fundada a todos los autores sería extremadamente engorroso y que es algo que se encuentra más allá de la capacidad de la gran mayoría de las revistas (Richardson, 1994: 11).

\section{Anonimato}

En muchas de las revistas jurídicas los encargados de seleccionar los artículos conocen su autoría. Se sostiene que los estudiantes editores se basan, con frecuencia, en la afiliación institucional y la reputación del autor al momento de escoger los artículos, remplazando así evaluaciones respecto a la calidad del artículo (Wise y otros, 2013: 8).

Distintas investigaciones empíricas han determinado que cuando no hay anonimato, los criterios para la selección del artículo pueden tener relación con el hecho de si el autor es muy influyente en su campo, si salda un vacío en la literatura existente, si el tema podría interesar al público jurídico general, si el autor ha publicado con frecuencia en revistas jurídicas prestigiosas, y si el autor trabaja en una universidad con renombre (Christensen y Oseid, 2007: 183).

Estas investigaciones también sugieren que la evaluación de los artículos examinados sin anonimato hace que sea más difícil para las mujeres, los extranjeros y los académicos más jóvenes sin antecedentes suficientes para publicar en estas revistas (Gingerich, 2009: 270-271). Además, otras investigaciones han llegado a la conclusión de que la calidad editorial no se ve afectada por la adopción de una política de anonimato en la revisión (Gingerich, 2009: 276).

\section{Valor pedagógico}

En opinión de algunos, pese a que la experiencia editorial continúa siendo un antecedente considerado como meritorio, el valor pedagógico de las revistas jurídicas ha disminuido significativamente con el correr de los años (Rotunda, 1987: 5). Esta 
disminución se explicaría a raíz de la abrumadora cantidad de trabajos recibidos, el aumento en la complejidad de tareas periféricas como la selección de nuevos miembros y la disminución del grado de involucramiento del personal de la facultad en las revistas. Por otro lado, el trabajo en las revistas jurídicas consume muchísima energía, algo que condiciona el tiempo dedicado al estudio en los estudiantes editores y los obliga, en muchos casos, a abandonar cursos (Wise y otros, 2013: 20).

No voy a detenerme a examinar el acierto de cada una de estas críticas, aunque pienso que sí son correctas aquellas formuladas en relación con el elitismo, los grupos desaventajados, la demora y falta de fundamentos ante el rechazo y el anonimato. Frente a este escenario, entiendo que Lecciones y Ensayos cuenta con algunas ventajas, por ejemplo, que ofrece a los autores un sistema de referato con garantía de anonimato para resguardar la imparcialidad del proceso. Además, existen restricciones y desincentivos para la publicación de artículos de los propios estudiantes editores en la revista. Por otra parte, no utiliza un sistema tan demandante como el Bluebook, sino que opta por un sistema de citación que es extremadamente sencillo y fácil de editar. Adicionalmente, existe un Consejo Asesor compuesto por académicos formados encargados de brindar un asesoramiento continuo. También manifiestan que para cada artículo existe una pluralidad de dictámenes escritos en los que se funda su aprobación o rechazo y, finalmente, para participar de la revista las calificaciones del estudiante son irrelevantes (aunque sí lo son para acceder a determinados cargos electivos), además de que el ingreso es irrestricto.

\section{Educación democrática para la educación jurídica}

Ahora quiero referirme a algunas perspectivas que pueden ser incorporadas a los debates que han tenido lugar. Suele decirse que uno de los verdaderos propósitos de las revistas jurídicas dirigidas por estudiantes es el de enseñarles a escribir, a editar y a pensar críticamente (Stracher, 2007-2008: 360; Harper, 1998: 1.272). Se añade que, como toda revista jurídica, contribuye también al desarrollo y a la difusión del derecho, puede ser utilizada como herramienta didáctica para la tarea docente, fortalece la memoria colectiva y preserva un registro de la cultura jurídica (Pugliese, 2014: 108). Por otra parte, alienta el debate doctrinario y, por tanto, efectúa un control sobre la tarea de jueces y otros funcionarios públicos. Sin embargo, tal vez hay algo más. Si los estudiantes con los propósitos comentados solo se encargaran de editar y evaluar los trabajos, sospecho que probablemente muchos de quienes participan en la revista dejarían de hacerlo (Mermin, 2004: 618).

En el caso del estudio escogido, las decisiones son tomadas por todos los integrantes del Consejo de Redacción compuesto por estudiantes de grado, los que una vez por semana se reúnen para debatir. Es importante precisar que no existen representantes o delegados, es decir, que la deliberación es directa. Los cargos electivos son 
ejecutivos y se encargan de llevar a cabo las decisiones surgidas del cuerpo editorial reunido en el pleno. Las normas que restringen el campo decisorio se reducen a unas pocas resoluciones del Consejo Directivo de la Facultad, ${ }^{1}$ con un grado alto de indeterminación en su contenido, y a la necesaria coordinación con el Departamento de Publicaciones, ya que la revista se encuentra bajo su órbita. De esta forma, queda un enorme margen de acción en torno a la selección de los trabajos que se reciben para ser publicados y a la gestión editorial.

La revista se convierte, así, en una de las actividades más comunales y disruptivas en el entorno de la enseñanza universitaria, una actividad capaz de fomentar la camaradería, la cooperación y un refugio con cierta autonomía respecto al resto de la estructura vertical de la universidad (Rosenkranz, 1988: 867-870). ${ }^{2}$ El propósito de este apartado es sostener que Lecciones y Ensayos es un nicho, muchas veces inexplorado, de la educación democrática. La revista constituye un terreno para estimular una educación no tradicional y que no solo pretende estar caracterizada por el horizontalismo, sino también por la deliberación en cooperación. Asimismo, es un espacio que antagoniza con el sistema de gestión característico de la educación superior. De algún modo, la revista es un espacio que pretende asegurar que la contribución de la institución educativa a la vida democrática no se reduzca a la acumulación de conocimientos, sino al ejercicio permanente de un debate crítico (Fiss, 2012: 271) capaz de enriquecer tanto la educación jurídica como la educación cívica de los estudiantes. ${ }^{3}$

Con esto presente, la revista puede funcionar a través de una dinámica democrática mucho más robusta de la que estamos habituados. Supone una educación que es democrática, pero que también es para la democracia (Mattern, 1997: 510). Por una parte, tenemos a la educación democrática, por su pretensión de horizontalismo y deliberación, y por su capacidad a veces solo potencial de tomar decisiones bajo estas condiciones. Por otra, está la educación para la democracia, por la posibilidad de desarrollar aptitudes para el examen crítico, la argumentación y la persuasión en el debate público comunitario, además de la tolerancia y el respeto por la autonomía del otro en un espacio de discusión que busca ser igualitarista. Todo esto supone, como se dijo, un modo no tradicional de fortalecer tanto la educación jurídica como la educación cívica de los estudiantes que participan del cuerpo editorial de la revista.

1. Resoluciones $2166 / 87$ del 29 de julio de 1987, 1205/94 del 16 de agosto de 1994 y 3949/o7 del 10 de abril de 2007 (la primera solo parcialmente vigente) del Consejo Directivo de la Facultad de Derecho de la Universidad de Buenos Aires. Disponible en bit.ly/2G6MybQ.

2. Existen modelos que pretenden incorporar algunas de estas notas en sus estructuras. Por ejemplo, el UC Irvine Law Review se editó por primera vez en 2011 y en sus orígenes apostó por innovar con un cuerpo editorial horizontal, en el que se ensayó un sistema de rotación en la asignación de tareas.

3. Si bien es un aspecto en el que no ahondaré, es curiosa la transformación que tiene lugar en el orden de jerarquías cuando son los estudiantes los que terminan evaluando las producciones de los docentes. 


\section{Educación jurídica}

En la Real Academia Carolina de Jurisprudencia en Charcas e incluso en la Academia de Jurisprudencia de Buenos Aires, el derecho era estudiado como una práctica argumentativa en la que se alentaba a los estudiantes a desarrollar aptitudes para el debate y el razonamiento (Böhmer, 2014: 386). Entre sus lecturas se encontraban las obras de aquellos filósofos políticos, como Rousseau, cuyas teorías tenían aptitud para operar contra el statu quo. Entre finales del siglo XVIII y principios del siglo XIX, este clima de debate propició el cuestionamiento de lo que sucedía en Latinoamérica y, en especial, de las autoridades políticas de aquel entonces. Por ejemplo, varios de los que se formaron en la Real Academia Carolina de Jurisprudencia en Charcas luego participaron activamente en las corrientes independentistas latinoamericanas. Se trataba, de todos modos, de un modelo educativo con una diversidad escandalosamente baja en lo que tiene que ver con la población universitaria, tanto en términos de género como de diversidad étnica o pertenencia de clase (Bergoglio, 2009: 14).

A finales del siglo XIX, y con la sanción del Código Civil, la educación legal fue reformada en la Argentina para fortalecer la legitimidad de las nuevas autoridades políticas. De esta forma, se abandonó la práctica argumentativa, se empezó a enseñar dogmática, se recurrió a modelos muy similares al formalismo jurídico y se exigió la repetición de textos de memoria (Böhmer, 2012: 14-15; Spector, 2008: 10). Se trató de un fenómeno que se desarrolló en Argentina y en gran parte de América Latina. De hecho, actualmente se mantiene en muchos casos.

En líneas generales, suele consistir en una enseñanza del derecho ritualista, acrítica, pretendidamente neutral, insularizada por encontrarse desapegada de la realidad social y con un predominio de la cátedra magistral con docentes de una dedicación parcial. Además, tiene relación con una retención memorística exacerbada a través de la repetición de códigos y leyes, un currículo enciclopédico, una insuficiente investigación académica y una marcada indiferencia respecto al aporte de las ciencias sociales. Sin duda, es una forma de enseñar que consolida un perfil de egresado con poca conciencia acerca del papel que al derecho le compete en el mantenimiento de las condiciones que caracterizan a nuestras sociedades. Por otra parte, también está relacionado a una forma de enseñar que reproduce, en gran parte, algunos de los aspectos más problemáticos de la tradición continental, pero que se ven agravados en nuestro continente por una profesión académica débil.

Usualmente, la única meta docente es transmitir la mayor cantidad de la legislación que conforma el derecho positivo argentino y la única meta estudiantil consiste en repetirla en los exámenes. Seis años de repetición de textos es, en muchos casos, la única actividad requerida por el Estado Nacional para quienes tienen consigo el monopolio de ser jueces y patrocinar a quienes desean acceder a la justicia (Böhmer, 2003; Böhmer, 2008: 369). 
En la actualidad, algunas investigaciones empíricas han concluido que este modelo ha conducido a que los estudiantes de abogacía posean una capacidad argumentativa de baja complejidad, ya que lo hacen con pocos elementos ajenos a la repetición de la ley vigente. Además, utilizan un vocabulario con pretensión de precisión técnica, pero no alcanzan a articularlo con sofisticación (Lista y Begala, 2004: 166-167). $\mathrm{Al}$ momento de tener que resolver un problema suelen adoptar una actitud de confrontación y no de conciliación, lo que evidencia una predisposición litigiosa (Lista y Begala, 2004: 167). Quizás, se podría esgrimir que esta preparación inadecuada repercute sobre la capacidad de concretar algún aporte significativo en el campo social. La experiencia en América Latina muestra que los abogados están mucho menos comprometidos con el cambio social de lo que se había creído en un principio y que, en consecuencia, el derecho es capaz de ser un poderoso multiplicador del statu quo (Duque Quintero, Quintero y Duque, 2014: 72).

Es cierto que, a partir de la sistemática violación de derechos en América Latina, en las últimas décadas ha ocurrido un progresivo y necesario giro en favor de una enseñanza del derecho que empieza a dejar atrás el énfasis memorístico para concentrarse en argumentaciones ancladas en la lógica de los derechos. Sin embargo, el ejercicio no siempre supera la idea de intentar incorporar, sin más precedentes, instrumentos internacionales a la realidad jurídica local, con métodos formalistas que se centran en repetir textos (Ronconi, 2017: 22-27).

Tal vez, Lecciones y Ensayos puede ser un elemento adicional capaz de antagonizar con este modelo formalista en el que predomina el uso de la memoria y la confrontación, ya que busca ser un espacio de permanente deliberación en cooperación, donde sus participantes argumentan y persuaden. $\mathrm{Al}$ abordar distintos temas del derecho o temas como la gestión editorial y la selección de los artículos en un contexto de discusión abierta, los estudiantes que participan del comité editorial se alejan del sistema tradicional de enseñanza del derecho. Se podría vislumbrar, entonces, una recuperación de la práctica argumentativa que existía en la región previo al fenómeno de la codificación.

\section{Educación cívica}

La historia de América Latina se encuentra surcada por el autoritarismo. En este contexto, Lecciones y Ensayos puede ser un instrumento más de formación para la democracia. Hace varias décadas que desde la filosofía de la educación se puntualiza la importancia de construir un perfil democrático en los estudiantes, especialmente durante la educación inicial y media, ahora, ¿por qué no hacerlo también en las familias y en los trabajos? (Gutmann, 1999: 91). Así, se sostiene que los estudiantes deben aprender no solo las habilidades que les permitan presentar exitosamente sus puntos de vista en el proceso democrático, sino también las aptitudes que los hagan toleran- 
tes tanto a las diferencias como a los resultados derivados de este proceso (Marks, 1988: 1143; Yudof, 1989: 42).

No obstante, parece razonable sostener que tal educación cívica no puede limitarse solo a los primeros años de educación formal. Además, es difícil enseñar con eficacia si no existe la posibilidad de emplear, en forma directa, lo que se enseña. La educación para la democracia es puesta a prueba cuando se puede continuar aprendiendo democracia haciendo democracia (Grady, 1990: 149).

En efecto, al participar en situaciones reales de deliberación, es decir, con poder de decisión, sin representantes y en un pie de igualdad, los estudiantes aprenden algunas de las habilidades y los valores necesarios para la participación en una sociedad democrática (Samuelsson y Bøyum, 2015: 79). El experimentalismo democrático permite que se desarrollen capacidades comunicativas relevantes (Englund, 2000: 309) y se estimule el empleo de la innovación y la creatividad en la búsqueda de mejores soluciones, porque la construcción de instituciones democráticas no tiene un punto de conclusión, sino que merece ser repensada en forma continua y colectiva (Bartenberger, 2015: 5). En este proceso, los estudiantes deben articular razones que puedan ser entendidas y aceptadas por los demás para poder construir consensos, abandonando así la idea de que la democracia es un mero espacio donde determinados intereses fijos compiten a través de mecanismos de una sumativa de preferencias (Samuelsson y Bøyum, 2015: 76-79). Así, puede ser mucho más que la votación basada en un criterio de mayorías: un proceso colectivo de deliberación intensa basado en una participación igualitaria (Bartenberger, 2015: 6).

El involucramiento en este tipo de debates no solo es una herramienta pedagógica (Elgueta Rosas y Palma González, 2014; González, 2013; Martín, Paoloni y Rinaudo, 2016; Mastache y Devetec, 2017; Mateo y Vlachopoulos, 2013; Osorio, 2013), sino que también tiene un componente moral nada desdeñable. Haber participado en un pie de igualdad de un proceso deliberativo a través del cual se tomó una decisión determinada provoca una mayor responsabilidad por parte del participante; una posible inclinación a respetar la mirada de sus pares y a tolerar la decisión colectiva (Bellamy, 2013: 15).

Todo esto también puede implicar tener mayores posibilidades de exigir, en el futuro, participar en los espacios que históricamente han sido reservados para unos pocos. En ese sentido, las exigencias democráticas no deben residir solo en los grandes centros de poder. No podemos pretender que las instituciones más relevantes para la comunidad sean fuertemente democráticas cuando las de menor envergadura poseen exigencias casi exiguas en esta materia. Por otro lado, los poderes del Estado no son las esferas exclusivas ni primarias de producción política del derecho ni del ejercicio de las libertades políticas. Buena parte del diálogo con consecuencias normativas ocurre fuera de estos principales canales formales de política electoral y política legislativa (Michelman, 1988: 1.531). Los funcionarios públicos pueden mos- 
trarse autoritarios, entre otros tantos motivos, porque no están habituados a prácticas de cierta intensidad democrática, sino expuestos durante toda una vida a las órdenes surgidas de distintas jerarquías.

La práctica deliberativa que puede desarrollarse en la revista permite a los estudiantes que evalúen, desde una perspectiva crítica, el modo en que funcionan las instituciones públicas en la actualidad (Hanson y Howe, 2011: 7). Así, se propicia un antagonismo con los modelos tradicionales. Es posible generar un contraste entre una práctica que se esfuerza por fortalecer la deliberación, la horizontalidad y otras que, muchas veces, están caracterizadas por el autoritarismo, el verticalismo y la participación de minorías privilegiadas en la toma de decisiones. La experimentación y la diversidad en los modos en que se administran los espacios públicos enriquecen el modo en que se toman las decisiones colectivas (Palma y Santos, 2015; Vélaz y De Paz Higuera, 2010; Pérez Perdomo, 2018). Es por eso mismo que, en muchos casos, es un despropósito cuando se pretende importar réplicas de lo que es la organización del poder de los gobiernos a la realidad de la revista.

Por otro lado, podría afirmarse que, en los hechos, la revista no es o no ha sido siempre un espacio caracterizado por estas notas y que, como en tantos otros sitios, abundan patrones de conducta basados en hegemonías que oprimen o en estrategias a través de las cuales quienes la integran persiguen la acumulación de poder o de réditos personales.

Es importante mencionar que los procesos que pretenden ser deliberativos no son inmunes a los comportamientos estratégicos (Hanson y Howe, 2011: 7). En la revista existen relaciones de poder, gestos autoritarios y cálculos de intereses, pero todo esto no afecta en nada la pretensión de avanzar hacia modelos a través de los cuales se intenten exponer estas relaciones a la deliberación pública, asumiendo esta última como un posible ideal para la toma de decisiones colectivas. Si bien puede existir autoritarismo, Lecciones y Ensayos es un espacio fértil para estimular un modelo opuesto: apostar por respuestas todavía más democráticas frente al comportamiento estratégico, un escenario no exageradamente idealizado que a veces puede ser muy ruidoso y en el que sus integrantes discuten con vehemencia (Habermas, 2005: 346 y 528; Waldron, 1999: 304-305).

\section{Posibles desafíos para el futuro}

En esta última parte del trabajo la propuesta consiste en señalar algunos de los desafíos para el futuro. Estimo que pueden ser de utilidad para aquellos que deseen involucrarse en los debates actuales en torno a las revistas jurídicas. Al mismo tiempo, corresponde aclarar que la reseña que se incluye a continuación constituye un conjunto de consideraciones muy generales de un observador externo, por lo que existen muchos aspectos que puedo estar pasando por alto de manera equivocada. 
Algunos de los más importantes desafíos podrían ser: el fortalecimiento de las lógicas democráticas como el deliberativismo horizontal en el cuerpo editorial, la transparencia en la información interna, una periódica rendición de cuentas a toda la comunidad académica y, también, la consolidación de procedimientos imparciales en el sistema de evaluación de los trabajos recibidos. Otro desafío podría ser el diseño de instrumentos capaces de aplacar los efectos negativos del recambio generacional permanente a través de buenos registros digitales y la estimulación de un Consejo Asesor activo, algo que en Estados Unidos ya es costumbre (Doyle, 2009: 187). Por otra parte, también sería deseable implementar la ampliación de las políticas de acceso abierto de los contenidos de la revista con la incorporación a más índices y catálogos, el robustecimiento de la versión digital de la revista como compromiso con el medio ambiente y el acceso masivo (evaluando la posibilidad de emplear programas como los desarrollados por Public Knowledge Project que agilizan la publicación del formato digital de la revista), y la revitalización del Consejo Latinoamericano de Publicaciones Jurídicas Estudiantiles por medio de nuevos acuerdos de cooperación con las revistas que lo integran. ${ }^{4}$

Las revistas jurídicas deberían ser capaces de ofrecer una vinculación entre las distintas áreas del derecho, que es algo que sucede en la realidad. El considerable aumento en el número de revistas especializadas tal vez podría llegar a operar en contra de esta posibilidad. Actualmente, los catálogos que condensan todo el material hemerográfico en un mismo espacio hacen que las revistas especializadas sean innecesarias. Los dramas sociales que existen en el mundo involucran consideraciones sobre distintas áreas del derecho. Pienso que es necesario contar con revistas que también se encuentren al servicio de los problemas legales más acuciantes surgidos de la extrema desigualdad, la debilidad de las instituciones democráticas y la depredación ambiental que existe en nuestro continente, entre otros temas.

Por otro lado, aún no se cuenta con estudios empíricos que echen luz sobre, por ejemplo, la incidencia de la experiencia editorial en los estudiantes, la composición del cuerpo editorial o la cantidad de estudiantes que han participado de la revista a lo largo de los años (Gordon, 1994: 543). Desde de la revista, quizás se pueda alentar el empleo de espacios de investigación con estos fines, sin embargo, incluso sin estudios de esta naturaleza, podría evaluarse la posibilidad de instrumentar acciones afirmativas y reconsiderar las exigencias establecidas para acceder a los cargos electivos con el fin de aplacar el elitismo y alentar la participación de los grupos históricamente marginados. Es preciso encontrar soluciones creativas que den con una alternativa capaz

4. En 1988 se llevó a cabo el Primer Encuentro Latinoamericano de Publicaciones Jurídica Dirigidas por Estudiantes, ocasión en la que se concretó la creación del Consejo Latinoamericano de Publicaciones Jurídicas Estudiantiles. Se trató de una iniciativa interesante que parece haber iniciado un debate que, según me parece, merece ser mantenido. 
de ofrecer una revista democrática, inclusiva y promotora de contenidos relevantes. No obstante, parece evidente la dificultad para evitar que en una sociedad con desigualdades estructurales el espacio no se convierta en un reproductor de las mismas.

Respecto a la posibilidad de mejorar los métodos de trabajo interno, en el New York Law School Law Review se contrató a un profesor «editor» a tiempo completo. Además de sus obligaciones como docente, se encarga de dictar un curso obligatorio para todos los estudiantes editores. En el curso no hay calificaciones, pero los estudiantes deben aprobarlo para obtener créditos por su participación en la revista. En la clase no solo se estudian diversas formas de investigación jurídica ni se discuten los propósitos y objetivos de la investigación, sino que también enseña cómo utilizar correctamente el sistema de citación (generalmente por medio del Bluebook) y cómo es recomendable escribir un artículo (Stracher, 2007-2008: 360). Con esto en mente, no parece una idea descabellada que algunas de las materias optativas que hoy se ofrecen en el Ciclo Profesional Orientado sean sobre investigación jurídica y estén orientadas a la gestión de las revistas. ${ }^{5}$

Asimismo, al encontrarse muy ocupados para cumplir con los plazos y manejar las operaciones del día, los estudiantes editores no siempre tienen la oportunidad de examinar sus roles en un sentido filosófico (Godsey, 1995: 87-88). Como consecuancia, parece determinante implementar un diálogo con la comunidad universitaria por medio de la organización de debates y jornadas de reflexión en espacios públicos y abiertos. Lo anterior no solo podría significar una oportunidad para examinarse de manera introspectiva, sino la posibilidad de nutrirse de los pareceres del resto de la comunidad. Además, podría actuar como un antídoto contra los efectos negativos del recambio generacional.

\section{Conclusión}

En la primera parte de este trabajo se mencionaron algunas de las discusiones en torno a las revistas jurídicas dirigidas por estudiantes de grado. Tales discusiones se centran, principalmente, en las distintas críticas manifestadas a estos espacios: el elitismo que suele caracterizarlos con la consiguiente afectación de los grupos más desaventajados de la comunidad universitaria, además de la baja utilidad del material publicado, el modo en que se lleva a cabo la selección de los artículos, la demora y falta de fundamentos ante un rechazo, la preservación del anonimato y la ausencia de valor pedagógico en la tarea editorial para los estudiantes. A partir de un caso de estudio concreto se intentó ampliar el espectro de discusiones que hoy se encuentran

5. El Ciclo Profesional Orientado se ubica en el último tramo de la carrera de Abogacía en la Facultad de Derecho de la Universidad de Buenos Aires. En él, los cursos suelen ser más pequeños y pretenden adoptar una modalidad similar a la de un seminario o taller. 
abiertas. De este modo, se destacaron dos elementos que parecen caracterizar estos espacios: i) una educación no tradicional que enfatiza la práctica argumentativa por sobre el método memorístico y enciclopédico; y ii) una educación que no es solo intensamente democrática, sino que se contrapone a los modelos a los que solemos estar habituados, algo que puede tener repercusiones positivas en nuestro desempeño como miembros de una comunidad política. Si bien abordé estos dos elementos en forma separada, es evidente que puede lograrse una valiosa retroalimentación entre ambos. Finalmente, se plantearon algunos desafíos que, en caso de asumirse, podrían potenciar la mejor versión de estos espacios.

\section{Referencias}

Allen, Danielle y Bernadette Maheandiran (2009). «You don't have to speak german to work on the German Law Journal': reflections on the value of being a student editor while being a law student». German Law Journal, 10: 1149-1167.

Álvarez Pertuz, Alicia, Omar Sandoval Fernández y Mildred Puello ScarPATTI (2018). «Estrategias pedagógicas para desarrollar competencias ciudadanas en estudiantes de Derecho». Revista Espacios, 39 (29): 1-6. Disponible en bit. ly/2rrbghr.

BArtenberger, Martin (2015) «John Dewey and David Graeber. Elements of radical democracy in pragmatist and anarchist thinking». Politix, 37: 4-9. DOI: 10.2139/ ssrn.2408571.

Bellamy, Richard (2013). «Constitutional democracy». En Michael T. Gibbons, Diana Coole, Elisabeth Ellis, Kennan Ferguson (editores), The Encyclopedia of Political Thought. Nueva Jersey: John Wiley \& Sons.

Bergoglio, María (2009). "Diversidad y desigualdad en la profesión jurídica: consecuencias sobre el papel del Derecho en América Latina». Via Iuris, 6: 12-28. Disponible en bit.ly/2PtC1Hg.

Bezanilla Albisua, María José, Manuel Poblete Ruiz, Donna Fernández Nogueira, Sonia Arranz Turnes y Lucía Campo Carrasco (2018). «El Pensamiento Crítico desde la Perspectiva de los Docentes Universitarios». Estudios Pedagógicos, 44 (1): 89-113. Disponible en bit.ly/2PssEsW.

BÖHMER, MARTín (2003). «Igualadores retóricos: las profesiones del derecho y la reforma de la justicia en la Argentina». Disponible en bit.ly/2E6cngz.

-. (2008). «Igualadores y traductores. La ética del abogado en una democracia constitucional». En Marcelo Alegre, Roberto Gargarella y Carlos Rosenkrantz (coordinadores), Homenaje a Carlos S. Nino (pp. 353-371). Buenos Aires: La Ley.

-. (2012). «Ensayo sobre la necesidad de consistencia pedagógica en el derecho argentino». Pensar en Derecho, o: 11-21. Disponible en bit.ly/2ElvUDW.

-. (2014). «Legal education Reform: How the Academy at Chuquisaca Forged 
Argentina's Founding Elite». Journal of Legal Education, 63: 373-404. Disponible en bit.ly/2zNN7lc.

CARRILlo, Isabel (2011). «La educación en valores democráticos en los manuales de la asignatura Educación para la Ciudadanía». Revista de Educacion, número extraordinario: 137-159. Disponible en bit.ly/2SDdaTq.

Casquero Oyarzabal, Oskar, Ramón Ovelar Beltrán, Jesús María Romo UriarTE y Manuel Benito (2014). «Entornos de aprendizaje personales, educación superior y analítica del aprendizaje: un estudio sobre los efectos de la multiplicidad de servicios en las redes personales de estudiantes universitarios». Cultura y Educación: Culture and Education, 26 (4): 696-738. Disponible en bit.ly/2BXxjOu.

Christensen, Leah y Julie Oseid (2007). «Navigating the law review article selection process: an empirical study of those with all the power-student editors». South Carolina Law Review, 59: 175-224. Disponible en bit.ly/2QIX79b.

Closen, Michael y Robert Dzielak (1996). «The History and Influence of the Law Review Institution». Akron Law Review, 30 (1): 15-53. Disponible en bit.ly/2Eas3so.

Cоттоn, Natalie (2006). «The competence of students as editors of law reviews: a response to Judge Posner». University of Pennsylvania Law Review, 154: 951-982. Disponible en bit.ly/2rrRe27.

Delgado Cintrón, Carmelo (1982). «Los fundadores de la Revista Jurídica». Revista Jurídica de la Universidad de Puerto Rico, 51: 1-9.

De Mello, Daniel Massimino y Danielle Anne Pamplona (2015). «Saberes» de Morin na educação jurídica: Caminhos à educação para o desenvolvimento sustentável». Opción, 31 (3): 446-469. Disponible en bit.ly/2G5KPDD.

De la Fuente Arias, Jesús, José Manuel Martínez, Francisco Peralta y Ana Belén GARCía (2010). «Percepción del proceso de enseñanza-aprendizaje y rendimiento académico en diferentes contextos instruccionales de la Educación Superior». Psicothema, 22 (4): 806-812. Disponible en bit.ly/2BZLEKe.

Doyle, John (2009). «The law reviews: do their paths of glory lead but to the grave?». Journal of Appellate Practice and Process, 10 (1):179-208. Disponible bit.ly/2PsbA4G.

Duque Quintero, Sandra, Marta Quintero y Derfrey Duque (2014) «Sobre una conceptualización de la popularización del derecho en la educación superior». Zona próxima, 21: 66-76. Disponible en bit.ly/2BZuuwu.

EDWARds, Harry T. (2014). «Another look at Professor Rofell's goodbye to law reviews». Virginia Law Review, 100: 1483-1511. Disponible en bit.ly/2SE8DQF.

Elgueta Rosas, María Francisca y Eric Eduardo Palma González (2014). «Una propuesta de clasificación de la clase magistral impartida en la Facultad de Derecho». Revista Chilena de Derecho, 41 (3): 907-924. Disponible bit.ly/2QM3Hfo.

ENGLUND, Tomas (2000). «Rethinking democracy and education: towards an education of deliberative citizens». Journal of Curriculum Studies, 32 (2): 305-313. DOI: $10.1080 / 002202700182772$. 
FIss, Owen (2012). «La misión democrática de la Universidad». Academia: Revista sobre Enseñanza del Derecho de Buenos Aires, 10 (20): 269-286. Disponible en bit. ly/2G8JwE5.

FOWLER, Thomas (2001). «Law reviews and their relevance to modern legal problems». Campbell Law Review, 24 (1): 44-52. Disponible en bit.ly/2BZzKjB.

García Jiménez, Enrique y Rocío Lorente García (2017). «De receptor pasivo a protagonista activo del proceso de enseñanza-aprendizaje: redefinición del rol del alumnado en la educación superior». Opción, 33 (84): 120-153. Disponible bit. ly/2Pu5Pno.

GAvA, John (2002). «Law reviews: good for judges, bad for law schools?». Melbourne University Law Review, 29: 560-579. Disponible en bit.ly/2L6iuvM.

GINGERICH, Jonathan (2009). "A call for blind review: student edited law reviews and bias». Journal of Legal Education, 59: 269-278. Disponible en bit.ly/2SboeAd.

Godsey, Mark (1995). "The myth of meritocracy, and the silencing on minority voices: the need for diversity of America's law reviews». Harvard Blackletter Law Journal, 12: 59-91. Disponible en bit.ly/2EiReKh.

Gómez, Cristina, Estrella Fernández, Estrella, Rebeca Cerezo y José NúÑEz (2018). «Dificultades de aprendizaje en educación superior: un reto para la comunidad universitaria». Publicaciones, 48 (1): 63-80. Disponible en bit.ly/2BZABAP.

GonzÁlez Galván, Jorge (2013). «Educación jurídica, investigación y derechos humanos inteligentes». Boletín Mexicano de Derecho Comparado, 46 (137): 499-527. Disponible en bit.ly/2SEPXjP.

Gordon, Wendy (1994). "Counter-manifesto: student-edited reviews and the intellectual properties of scholarship». University of Chicago Law Review, 61: 541-551. Disponible en bit.ly/2PpAfa4.

Grady, Robert C. (1990). «Workplace Democracy and Possessive Individualism». The Journal of Politics, 52 (1): 146-166. Disponible en bit.ly/2QkFPQn.

Grosenick, Evelyn (2013). "In defense of law reviews: a response to Megan S. Knize's article, the pen is mightier: rethinking the 'gladiator' ethos of student-edited articles». McGeorge Law Review, 45: 305-318. Disponible en bit.ly/2G4BagU.

Gutmann, Amy (1999). Democratic education. Nueva Jersey: Princeton University Press. Habermas, Jürgen (2005). Facticidad y validez. Madrid: Trotta.

Hanson, Jarrod y Kenneth Howe (2011). "The Potential for Deliberative Democratic Civic Education». Democracy \& Education, 19 (1): 1-9. Disponible en bit. ly/2G4zWSQ.

HARPer, James (1998). «Why student-run law reviews». Minnesota Law Review, 82: 1261-1295. Disponible en bit.ly/2Ek9QcF.

KLINGER, Michael (2015). «Escape from the navel-gazing academy: a modest proposal for student-edited legal scholarship». UC Irvine Law Review, 5: 179-195. Disponible en bit.ly/2Qmr6UW. 
KNIZE, Megan (2013). "The pen is mightier: rethinking the 'gladiator' ethos of student-edited law reviews». McGeorge Law Review, 44: 309-337. Disponible bit. ly/2UtlSoQ.

Kyмlicka, Will (1995). Filosofía política contemporánea: Una introducción. Barcelona: Ariel.

Lista, Carlos y Silvana Begala (2004). «La presencia del mensaje educativo en la conciencia de los estudiantes: resultados de la socialización en un modelo jurídico dominante». Academia: Revista sobre Enseñanza del Derecho de Buenos Aires 2: 147-171. Disponible en bit.ly/2E6Wrnr.

MARKs, Jonathan (1988). «Reseña de Democratic education de Amy Gutmann». Michigan Law Review, 86: 1140-1147.

Martín, Rocío, Paola Paoloni y María Cristina Rinaudo (2016). «Percepciones y expectativas sobre el contexto de aprendizaje. Un estudio con alumnos de Educación Superior». Revista Interamericana de Psicología, 49 (2): 213-221. Disponible en bit.ly/2zOAoyI.

Martineau, Bradley (2001). «The future of law reviews and legal journals from a student editor's perspective». University of Pittsburgh Journal of Technology Law and Policy, 2: 1-14. Disponible en bit.ly/2B1pkOE.

Mastache, Anahi y Ronald Devetac (2017). «La formación jurídica: una mirada desde una didáctica analítica y reflexiva». Academia: Revista sobre Enseñanza del Derecho de Buenos Aires, 15 (30): 153-18o. Disponible bit.ly/2Ed6k3X.

Mateo, Joan y Dimitrios Vlachopoulos (2013). «Reflexiones en torno al aprendizaje y a la evaluación en la universidad en el contexto de un nuevo paradigma para la educación superior». Educación XXI, 16 (2): 183-208. Disponible en bit. ly/2QlGghF.

Mattern, Mark (1997). «Teaching Democratic Theory Democratically». PS: Political Science and Politics, 30 (3): 510-515.

Mermin, Jonathan (2004). «Remaking law review». Rutgers Law Review, 56: 603-623. Disponible en bit.ly/2Uu4vUR.

Michelman, Frank (1988). «Law's republic». Yale Law Journal, 97: 1.493-1.537. Disponible en bit.ly/2QiSjbb.

Movsesian, Mark (2005). «Introduction: A good idea». Hofstra Law Review, 33 (4): 1.121-1.122. Disponible en bit.ly/2E79PYO.

MulLins, Jennifery y Nancy LeONG (2011). «The persistent gender disparity in student note publication». Yale Journal of Law \& Feminism, 23 (2): 385-444. Disponible en bit.ly/2rpRzSN.

Nichols, Phil (1987). «A student defense of student edited journals: in response to Professor Roger Cramton». Duke Law Journal, december: 1.122-1.137. Disponible en bit.ly/2B1At28. 
Osorio, Aura (2013). «Hacia la excelencia en la educación jurídica». Vniversitas, 126: 11-14. Disponible en bit.ly/2QJYRPm.

Palma Gonzalez, Eric Eduardo y Antonia Santos (2015). «Derechos económicos, sociales y culturales como límites a las políticas públicas: El caso del derecho a la educación en Chile». Revista Derecho del Estado, 34: 237-254. Disponible en bit. ly/2GgoTTI.

Pérez Perdomo, Rogelio (2018). «De Harvard a Stanford. Sobre la historia de la educación jurídica en los Estados Unidos». Boletín Mexicano de Derecho Comparado, 51 (151): 313-358. Disponible en bit.ly/2RLsQUo.

Posner, Richard (1995). «The Future of the Student-Edited Law Review». Stanford Law Review, 47: 1131-1138. Disponible en bit.ly/2rqOCBo.

Pugliese, María Rosa (2007). «Las revistas jurídicas». En Víctor Tau Anzoátegui (coordinador), Antología del pensamiento jurídico argentino (1901-1945) (pp. 2972). Tomo II. Buenos Aires: Instituto de Investigaciones de Historia del Derecho.

-. (2014). «Las revistas jurídicas en la Argentina en la primera mitad del siglo XX». Revista de Historia del Derecho, 47: 105-148. Disponible en bit.ly/2RL4eeu.

-. (2015). «Las revistas jurídicas: un instrumento didáctico para el estudio de la evolución del derecho en la Argentina». Academia: Revista sobre Enseñanza del Derecho de Buenos Aires, 25: 75-107. Disponible en bit.ly/2UsIXso.

Richardson, David M (1994). «Improving the law review model: a case point». Journal of Legal Education, 44: 6-12.

Rodell, Fred (1936). «Goodbye to Law Reviews». Virginia Law Review, 23: 38-45. Disponible en bit.ly/2L3rTV9.

RonCONI, Liliana (2017). «La enseñanza en derechos humanos en las facultades de derecho en la Argentina: desafíos pendientes». Revista Pedagógica Universitaria y Didáctica del Derecho, 4 (1):5-37. Disponible en DOI: 10.5354/0719-5885.2017.46249.

Rosenkranz, E. Joshua (1988). «Law review’s empire». Hastings Law Journal, 39 (4): 859-926. Disponible en bit.ly/2GgYHLk.

Rotunda, Ronald (1987). «Law reviews - The extreme centrist position». Indiana Law Journal, 62 (1): 1-11. Disponible en bit.ly/2ElE7I2.

SAmuelsson, Martin y Steinar Bøyum (2015). «Education for deliberative democracy: Mapping the field». Utbildning and Demokrati, 24 (1): 75-94. Disponible en bit. ly/2UuyufI.

Sloviter, Dolores (2002). «In praise of law reviews». Temple Law Review, 75: 7-12.

Sмiтн, Talibah-mawusi (2011). «The law and educational inequities: in other words, the dilemma of writing while black». Critical Studies Journal, 4 (2): 73-97. Disponible en bit.ly/2IhRfgk.

SPeCtor, Horacio (2008). «The academic study of law in Argentina». Cuadernos Unimetanos, 15: 6-15. Disponible en bit.ly/2BZDTnF. 
STRACHER, Cameron (2007-2008). «Reading, writing and citing: In praise of law reviews». New York Law School Law Review, 52: 349-370. Disponible en bit. ly/2PruCA.

STURM, Susan P. (1997). «From gladiators to problem-solvers: connecting conversations about women, the academy, and the legal profession». Duke Journal of Gender Law \& Policy, 4 (1): 119-147. Disponible en bit.ly/2zQJwD2.

Vélaz, Consuelo y Ana Belén de Paz Higuera (2010). «Investigar sobre el derecho, el deseo y la obligación de aprender en la sociedad del conocimiento». Revista de Educación, (1): 17-30. Disponible en bit.ly/2 $\mathrm{G} 4 \mathrm{~F} 7 \mathrm{Cg}$.

WALDRON, Jeremy (1999). Law and disagreement. Oxford: Oxford University Press.

Wise, Richard, Lucy S. McGough, James W. Bowers, Douglas P. Peters, Joseph C. Miller, Heather K. Terrell, Brett Holfeld y Joe H. NeAl (2013). «Do law reviews need reform? A survey of law professors, student editors, attorneys, and judges». Loyola Law Review, 59: 1-75. Disponible en bit.ly/2PrVEPX.

Yudof, Mark (1989). «Reseña de Democratic education de Amy Gutmann». Ethics, 99: 439-441.

\section{Sobre el autor}

Patricio Enrique Kenny es abogado por la Universidad de Buenos Aires, docente de la misma casa de estudios y de la Universidad de San Andrés. Su dirección de correo electrónico es: pkenny@udesa.edu.ar. El trabajo es consecuencia de una investigación realizada en 2015 y con revisiones de sus resultados en 2016 y 2018. Agradece especialmente a Francisco Sáez Zamora y a los dos árbitros anónimos que evaluaron esta publicación. 
La Revista Pedagogía Universitaria y Didáctica del Derecho (RPUDD) es una publicación científica semestral que contribuye a la reflexión multidisciplinaria sobre pedagogía universitaria y didáctica del derecho, para la formación y consolidación de esta área de investigación; así como a la difusión de prácticas innovadoras en la enseñanza-aprendizaje del derecho considerando el contexto nacional e internacional. Es una publicación electrónica internacional con una codirección entre Brasil y Chile.

\author{
DIRECTORA \\ María Francisca Elgueta Rozas \\ Universidad de Chile \\ DIRECTOR \\ Renato Duro Dias \\ Universidad Federal de Rio Grande, Brasil \\ SITIO WEB \\ pedagogiaderecho.uchile.cl \\ CORREO ELECTRÓNICO \\ rpedagogia@derecho.uchile.cl \\ LICENCIA DE ESTE ARTÍCULO
}

Creative Commons Atribución Compartir Igual 4.o Internacional

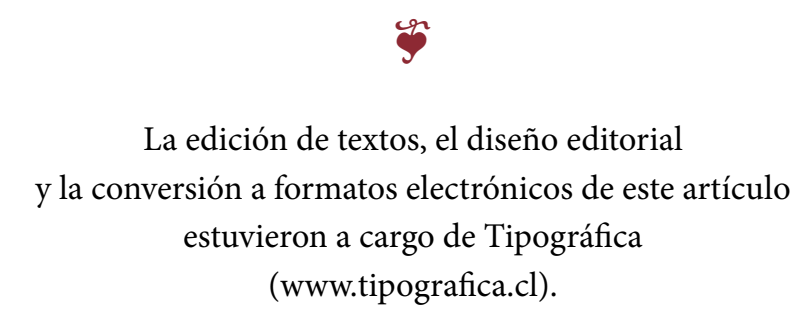

